\title{
Mechanical and genetic separation of aster- and midzone-positioned cytokinesis
}

Henrik Bringmann ${ }^{1}$

Max Planck Institute for Molecular Cell Biology and Genetics, Pfotenhauerstrasse 108, 01307 Dresden, Germany

\begin{abstract}
The mitotic spindle positions the cytokinesis furrow. The cytokinesis furrow then forms and ingresses at the site of the mitotic spindle, between the spindle poles. Two populations of spindle microtubules are implicated in cytokinesis furrow positioning: radial microtubule arrays called asters and bundled non-kinetochore microtubules called the spindle midzone. Here I will discuss our recent results that provided examples of how aster-positioned and midzone-positioned cytokinesis can be mechanically and genetically separated. These experiments illustrate how asters and midzone contribute to cytokinesis. ASS (asymmetric spindle severing) is a mechanical way to spatially separate the aster and midzone signals. In Caenorhabditis elegans embryos, asters and midzone provide two consecutive signals that position the cytokinesis furrow. The first signal is positioned midway between the microtubule asters; the second signal is positioned over the spindle midzone. Aster and midzone contribution can also be genetically separated. Mutants in spd-1 have no detectable midzone and are defective in midzone-positioned but not aster-positioned cytokinesis. Disruption of the function of LET-99 and the heterotrimeric G-proteins G0A-1/GPA-16 and their regulator GPR-1/2 causes defects in aster-positioned cytokinesis but not in midzone-positioned cytokinesis. In order to understand aster-positioned cytokinesis we have to understand how microtubule asters spatially control the activity of LET-99, GPR-1/2 and G0A-1/GPA-16 and how the activity of these G-protein pathway components control the assembly of a contractile ring.
\end{abstract}

\section{Mechanical separation of aster and midzone contribution to cytokinesis}

The mitotic spindle induces and positions the cytokinesis furrow, which then forms at the site of the spindle between the two spindle poles and ingresses to physically separate the two daughter cells. Microtubule asters and the spindle midzone have both been implicated in positioning the cytokinesis furrow. Microtubule asters are radial arrays of microtubules nucleated by the centrosome. The spindle midzone is an antiparallel microtubule array that forms at anaphase between the segregating chromosomes [1]. There are four extreme models as to how these two spindle substructures could contribute to furrow positioning. Two models assume a dominant mechanism and two assume a redundant mechanism: (1) asters dominate positioning of the cytokinesis furrow; (2) the spindle midzone dominates positioning of the cytokinesis furrow; (3) both the asters and the midzone contribute to one furrow-positioning signal; (4) both the asters and the midzone provide independent signals. The problem with resolving these four models is determining the relative contributions from the two different parts of the spindle.

The mitotic spindle is a symmetrical structure. A furrowpositioning cue from the asters would position the furrow midway between them. A midzone cue would position a furrow at the same place. In order to separate the contributions of

Key words: aster, cytokinesis, G-protein, microtubule, midzone. Abbreviations used: ASS, asymmetric spindle severing; RNAi, RNA interference. 1email bringman@mpi-cbg.de the midzone and the asters to cytokinesis, the two structures must be spatially separated. A spatial separation of the region midway between the asters and the spindle midzone would occur in an asymmetric spindle in which one half-spindle is longer than the other. Such asymmetry would displace the midzone toward one side of the spindle and thus no longer position it midway between the asters. Depending on the models presented above, one would expect the following results shown in Figure 1: Model 1, if the asters dominate positioning the cytokinesis furrow, the furrow will form midway between the asters; Model 2, if the spindle midzone dominates positioning the cytokinesis furrow, the cytokinesis furrow will form at the site of the spindle midzone; Model 3, if both the microtubule asters and spindle midzone contribute to a single positional signal for cytokinesis furrow formation, the furrow will be positioned in some intermediate location; Model 4, if both the microtubule asters and the spindle midzone contribute independent signals to position the cytokinesis furrow, one furrow will form midway between the asters and one furrow will form over the spindle midzone.

How can an asymmetric spindle be generated? At anaphase, before the position of the furrow is determined but after the formation of the spindle midzone, one aster is separated from its associated chromatin using an ultraviolet laser. This procedure is called ASS (asymmetric spindle severing). This procedure creates a cell with one isolated aster and the other aster still attached to the midzone. Cortical pulling forces move the asters to opposite poles of the cell, positioning the spindle 'midzone' roughly one-third of the 
Figure 1 Mechanical separation of aster- and midzone-positioned cytokinesis

The relative contributions of asters (green) and midzone (red) to cytokinesis is difficult to study in a wild-type spindle because of the inherent symmetry: The region midway between the aster centres and the spindle midzone coincide. Asymmetric spindle severing causes a spatial separation of asters and midzone, allowing testing of different models regarding the relative contributions of asters and spindle. After asymmetric spindle severing, two furrows form, a first furrow between the asters, and a second furrow over the spindle midzone, as in model 4.

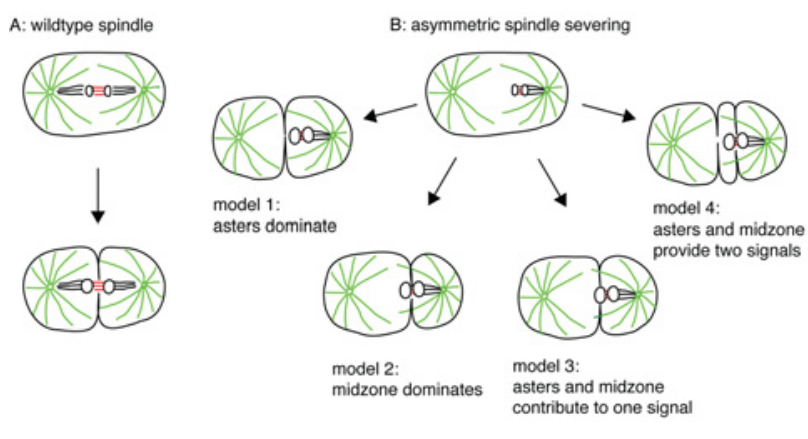

way along the aster-to-aster-distance, creating an asymmetric spindle. Following ASS, cytokinesis furrow ingression starts midway between the asters. However, the furrow does not complete midway between the asters: the furrow pauses and a second furrow forms at the cell cortex closest to the midzone. The two distinct furrows then meet and cytokinesis completes, forming two cells. Thus, two furrows are observed after ASS, first a furrow between the asters and then a second furrow directed towards the midzone. These experiments suggest that a cytokinesis furrow is specified by two consecutive signals derived from distinct structures of the mitotic spindle: first, the microtubule asters and secondly, the spindle midzone (model 4) [2].

\section{Genetic separation of aster and midzone contribution to cytokinesis}

A prerequisite for a complete understanding of cytokinesis is an inventory of all the proteins involved. Chemical mutagenesis screens, RNAi (RNA interference) screens and biochemical purification have identified proteins required for cytokinesis (for reviews, see $[3,4]$ ). Most of the proteins identified so far are required for both aster and midzone-positioned cytokinesis.

Microtubule plus ends from the asters contact the cortex, whereas the spindle midzone is located in the centre of the cell. A signal from the asters most likely depends on the contact between microtubules and the cortex whereas a signal from the midzone has to be transduced to the cortex. Thus one would expect that microtubule asters and the spindle midzone may use different mechanisms for furrow positioning and thus also may use different molecules. If this assumption is correct it should be possible to identify
Figure 2 Genetic separation of aster and midzone-positioned cytokinesis

This cartoon illustrates the principle of the spd-1 screen that was used to identify genes that are required for aster-positioned cytokinesis, but are dispensable for midzone-positioned cytokinesis. spd-1 mutants lack a spindle midzone (red) and midzone-positioned cytokinesis but can divide using aster-positioned cytokinesis (asters are green). RNAi of embryonic lethal genes was performed in spd-1 mutants and in wild-type. If the RNAi disrupts the function of an aster-postioned cytokinesis gene, wild-type embryos should divide but spd-1 embryos should not. Screening in the absence of a spindle midzone identified a role of LET-99, GPR-1/2 and GOA-1/GPA-16 in aster-positioned cytokinesis.

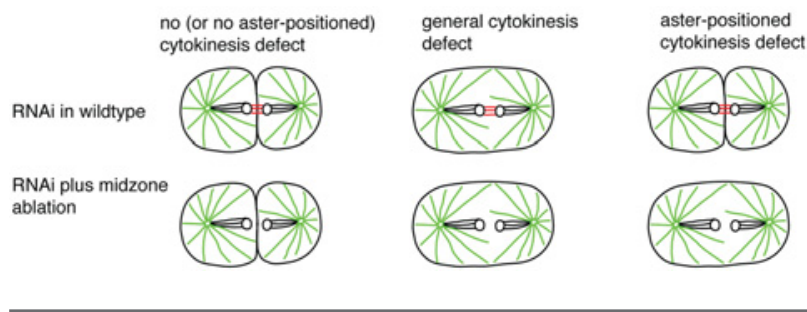

mutants that are defective in either midzone or asterpositioned cytokinesis. If both pathways are fully redundant, that means that both microtubule asters and the spindle midzone can induce and complete a cytokinesis furrow.

This idea can be tested in embryos lacking a functional spindle midzone. spd-1 mutant embryos lack a detectable midzone [5]. ASS analysis shows that $s p d-1$ mutants cannot form a midzone-positioned furrow. However, after ASS, the aster-positioned cytokinesis furrow forms and the cell divides [2]. Thus cells can cleave efficiently without a spindle midzone, as occurs in spd-1 mutants. Genes required for aster-positioned cytokinesis have been identified using an RNAi-based screen. The screen was perfomed for genes required for cytokinesis only in the absence of a spindle midzone. Figure 2 illustrates the principle of the screen. Genes were identified based on the assumption that embryos defective in aster-positioned cytokinesis will cleave in the presence of a spindle midzone, and will not cleave in the absence of a spindle midzone. The spindle midzone can be disrupted genetically using spd-1(oj5) mutant embryos or mechanically using a highly focused UV laser beam. The results from these studies suggest that, in the absence of a functional spindle midzone, GOA-1/GPA-16, GPR-1/2 and LET-99 are required for cytokinesis, indicating that these proteins are required for aster-positioned cytokinesis [6].

GOA-1/GPA-16, GPR-1 and LET-99 are proteins know to be involved in mitotic spindle positioning. In C. elegans, the mitotic spindle forms in the centre of the cell and at anaphase the spindle is displaced to the posterior by cortically anchored pulling forces. The number of force generators and thus the forces are higher in the posterior, explaining the displacement. The PAR proteins control these pulling forces [7]. GOA-1/GPA-16, GPR-1 and LET-99 are thought to act downstream of the PAR proteins to 
control spindle positioning [8-12]. In GOA-1/GPA-16- and GPR-1-depleted embryos, the spindle is not displaced to the posterior and the pulling forces are strongly reduced $[8,11,12]$. In LET-99-depleted embryos, the spindle does not centre, but forms in the posterior. The asters show vigorous rocking movements and it was suggested that pulling forces are increased in these embryos [9,13]. The involvement of spindle positioning genes in aster-positioned cytokinesis may mean that spindle positioning and aster-positioned cytokinesis are the same mechanical problem.

LET-99 contains a conserved DEP domain (domain present in Dishevelled, EGL-10, Pleckstrin). LET-99 localizes to the cortex in a band slightly posterior of the cell middle [10]. At anaphase, LET-99 localizes precisely at the site of cytokinesis furrow formation [6]. To show that the mitotic spindle positions LET-99, the mitotic spindle can be displaced at metaphase using a fine glass needle and the position of the band can be followed with a fluorescent LET-99 construct. In these embryos, the position of the band and the spindle does not coincide before anaphase, indicating that the spindle does not position cortical LET-99 at that cell cycle stage. At anaphase, however, the cortical LET-99 band moves to the site of the cortex overlying the spindle, indicating that the spindle positions cortical LET-99 at that stage [6]. This finding is consistent with the classic view that the cytokinesis furrow induces signals that are active at anaphase and not before [14].

GPR-1/2 is a G-protein regulator that acts through the redundant $\mathrm{G}_{\alpha}$ subunits GOA-1 and GPA-16 [8,11]. LET-99 and heterotrimeric G-proteins act in the same pathway [13]. GOA-1/GPA-16-depleted embryos form a LET-99 band that is visible until metaphase. However, at the time of cytokinesis furrow formation, the cortical distribution of LET-99 appears to be unrelated to the position of the cytokinesis furrow and the strict enrichment of cortical LET-99 at the site of the cytokinesis furrow disappears. Thus, the metaphase and the anaphase LET-99 bands appear to be genetically distinct [6].

GPR-1 localization is very different from LET-99 localization. At the time of furrow formation, GPR-1 is reduced at the poles and at the site of cytokinesis furrow formation. Thus, at the time of cytokinesis furrow formation, cortical GPR-1 localization can be described as two broad peaks flanking the site of furrow formation. The two peak maxima roughly correlate with the positions of the asters and the minimum between the two asters correlates with the site of cytokinesis furrow formation [6].

Depletion of LET-99 shows that LET-99 is required for the reduction of GPR-1 at the site of furrow formation. In the absence of LET-99, GPR-1 still localizes to asters and the cortex in let-99(RNAi) embryos. At the time of cytokinesis furrow formation, cortical GPR-1 is not excluded from the site of cytokinesis furrow formation. Instead, cortical GPR-1 is enriched at the site of furrow formation. Similar to LET99, the maximum of cortical GPR-1 coincides with the site of furrow formation. Thus, in the absence of LET-99, GPR-1 localization is inverted: instead of being reduced at the site of furrow formation, it is enriched at this site, similar to the localization of LET-99 in wild-type embryos. These data show that LET-99 and components of the G-protein pathway interdepend for their localization: LET-99 excludes GPR-1 from the site of cytokinesis furrow formation and the G-proteins localize LET-99 to the site of cytokinesis furrow formation [6].

What are the key questions for the future? The key problems are: how do microtubule asters spatially control the activity of LET-99, GPR-1/2 and GOA-1/GPA-16? And how does the activity of these G-protein pathway components cause the assembly of a contractile ring?

\section{References}

1 Rappaport, R. (1996) Cytokinesis in Animal Cells, Cambridge University Press, Cambridge

2 Bringmann, H. and Hyman, A.A. (2005) A cytokinesis furrow is positioned by two consecutive signals. Nature 436, 731-734

3 Eggert, U.S., Mitchison, T.J. and Field, C.M. (2006) Animal cytokinesis: from parts list to mechanisms. Annu. Rev. Biochem. 75, 543-566

4 Glotzer, M. (2005) The molecular requirements for cytokinesis. Science 307, 1735-1739

5 Verbrugghe, K.J. and White, J.G. (2004) SPD-1 is required for the formation of the spindle midzone but is not essential for the completion of cytokinesis in C. elegans embryos. Curr. Biol. 14, 1755-1760

6 Bringmann, H., Cowan, C.R., Kong, J. and Hyman, A.A. (2007) LET-99, GOA-1/GPA-16, and GPR-1/2 are required for aster-positioned cytokinesis. Curr. Biol. 17, 185-191

7 Grill, S.W., Gonczy, P., Stelzer, E.H. and Hyman, A.A. (2001) Polarity controls forces governing asymmetric spindle positioning in the Caenorhabditis elegans embryo. Nature 409, 630-633

8 Colombo, K., Grill, S.W., Kimple, R.J., Willard, F.S., Siderovski, D.P. and Gonczy, P. (2003) Translation of polarity cues into asymmetric spindle positioning in Coenorhabditis elegans embryos. Science 300, 1957-1961

9 Rose, L.S. and Kemphues, K. (1998) The let-99 gene is required for proper spindle orientation during cleavage of the $C$. elegons embryo. Development 125, 1337-1346

10 Tsou, M.F., Hayashi, A., DeBella, L.R., McGrath, G. and Rose, L.S. (2002) LET-99 determines spindle position and is asymmetrically enriched in response to PAR polarity cues in C. elegans embryos. Development $\mathbf{1 2 9}$, 4469-4481

11 Gotta, M., Dong, Y., Peterson, Y.K., Lanier, S.M. and Ahringer, J. (2003) Asymmetrically distributed $C$. elegans homologs of AGS3/PINS control spindle position in the early embryo. Curr. Biol. 13, 1029-1037

12 Srinivasan, D.G., Fisk, R.M., Xu, H. and van den Heuvel, S. (2003) A complex of LIN-5 and GPR proteins regulates $\mathrm{G}$ protein signaling and spindle function in C elegans. Genes Dev. 17, 1225-1239

13 Tsou, M.F., Hayashi, A. and Rose, L.S. (2003) LET-99 opposes G $\alpha /$ GPR signaling to generate asymmetry for spindle positioning in response to PAR and MES-1/SRC-1 signaling. Development 130, 5717-5730

14 Hiramoto, Y. (1956) Cell division without mitotic apparatus in sea urchin eggs. Exp. Cell Res. 11, 630-636

Received 7 January 2008

doi:10.1042/BST0360381 